\title{
Tension Viscerothorax In A Patient With Missed Traumatic Diaphragmatic Hernia. A Case Report.
}

\author{
Herbert Butana, Laurance Ntawunga, Desire Rubanguka, and Isaie Sibomana
}

\begin{abstract}
Background: Diaphragmatic hernias occurring post trauma are a challenge to diagnose early especially when they follow blunt trauma. Many of those diagnosed early occur in penetrating thoraco-abdominal trauma which necessitates emergency exploration where the diagnosis is picked. Rarity of traumatic diaphragmatic hernia coupled with poor sensitivity of easily available imaging modalities makes it a big challenge to pick up this potentially fatal pathology.

Case presentation: We present a rare case of tension viscerothorax in a young man who had presented to the emergency department at a provincial hospital of Rwanda three days before the second consultation where the chest $x$ ray was interpreted as normal and later as a pneumothorax before the diagnosis and treatment of tension viscerothorax could be made.

Conclusion: Viscerothorax is an elusive diagnosis which when missed can complicate to strangulation of hernia contents or tension viscerothorax which carry a high mortality.

Keywords - tension viscerothorax; trauma; diaphragmatic hernia; Rwanda.
\end{abstract}

\section{INTRODUCTION}

Post-traumatic diaphragmatic hernias are rare, but easily missed cases especially in blunt chest and abdominal trauma. The incidence of traumatic diaphragmatic injuries is estimated at $0.8 \%$ to $5 \%$ and males are more affected [1]. Unrecognized injuries carry significant morbidity and mortality varying between 30 and $60 \%$ but which is higher in tension viscerothorax [2] [3]. They commonly occur following motor vehicle accidents, falls and stab wounds. Poor sensitivity of initial imaging modalities for diaphragmatic injuries lead to delayed diagnosis and therefore high index of suspicion is paramount in diagnosis and prevention of fatal complications [2].

Patients become symptomatic when abdominal viscera herniate through diaphragmatic defect and depending on the size, tension viscerothorax may ensue with kinking of inferior vena cava and then reduction of venous return and cardiac output. The result is obstructive shock and therefore, tension viscerothorax should be considered in patients with obstructive shock who has prior history of chest or abdominal trauma [4].

Published on April 28, 2020

Herbert Butana, Surgery Department, Rwamagana Provincial Hospital, Rwanda.

(Corresponding e-mail: herbertbutana@gmail.com)

Ntawunga Laurance, Surgery Department, University of Rwanda.

(e-mail: nlisaaya@gmail.com)

Desire Rubanguka, Surgery Department, King Faisal Hospital, Rwanda. (e-mail: drubanguka@gmail.com)

Isaie Sibomana, Surgery Department, Kibuye Referral Hospital, University of Rwanda.

(e-mail: siibomana@gmail.com)
The herniated viscera are at risk of getting strangulated, perforated leading to sepsis and cardiovascular collapse. After stabilization of hemodynamic status which is difficult to achieve before removing the cause of obstructive shock, treatment is surgical with laparotomy or thoracotomy approaches, but laparotomy is preferred in acute settings to exclude associated intra-abdominal injuries [2].

This study aimed to present a case of tension viscerothorax in a patient with missed traumatic diaphragmatic hernia in a setting with limited radiologic diagnostic capacity in a rural provincial hospital in Rwanda who was successfully managed.

\section{CASE REPORT}

We present a 32 year old male who came in 3 days post the first contact at our emergency department. He presented with recent onset difficulty in breathing and chest pain. He reported being involved in a road traffic accident 3 days before (motor cycle to truck collision). He had no history of chronic respiratory diseases like asthma. He reported no body swelling and no allergy. Review of the chest X-ray done at the first contact revealed viscerothorax which had been interpreted as normal (Fig.1).

On physical examination he was breathless, sweating with respiratory rate of $38 \mathrm{bpm}, \mathrm{SPO} 2$ of $64 \%$ on room air and $90 \%$ on 10 liters of oxygen. There was no cyanosis and pulse rate was $130 \mathrm{bpm}, \mathrm{BP}$ of $103 / 56 \mathrm{mmhg}$ and temperature of $36.6^{\circ} \mathrm{C}$.

He was in severe respiratory distress with use of accessory respiratory muscles. Chest was asymmetric with right hemithorax bulging and left barely moving. Auscultation revealed a silent left hemithorax, but vesicular breath sounds on the right side. Dull percussion note on left side versus normal percussion note on right was noted and heart sounds were auscultated on the right side of chest.

Other systems examination were unremarkable

Urgent investigations done, FBC: WBC-15.5X10 $/ 1 /$; HB8.3g/dl; HCT- 24.7\%; MCV-91.8fL; PLT-294X10 $/ 1$

Abdominal ultrasound -no free fluid

Chest radiograph revealed, trachea deviation to the right, collapsed left lung, viscerothorax (collar sign), mediastinal shift to right and loss of left hemi-diaphragm outline (Fig.2). Supplemental oxygen, fluid resuscitation were started, an emergency laparotomy followed by chest tube thoracostomy were performed. 


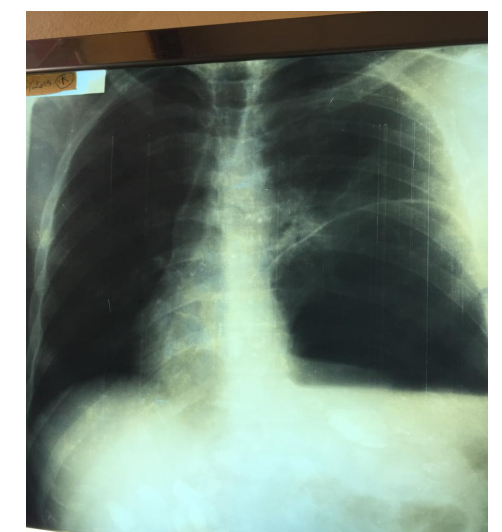

Fig.1: Chest radiography of patient presented. Initial chest radiography taken at the first consult interpreted as normal Xray.

Second chest X-ray misinterpreted as pneumothorax before the surgeon concluded on viscerothorax: Note partial collapse of left lung, loss of left diaphragmatic outline, intra thoracic bowel andmediastinal shift to the right.

Intra-operative findings: hemoperitoneum, $6 \mathrm{~cm}$ tear in left hemidiaphragm where small bowel, transverse colon, omentum and stomach were herniating through defect and viable (Fig.3, 4). Herniating contents were reduced, repair of the defect using nylon $\mathrm{N}^{0} 1$ continuous suture was performed and a chest tube was placed.

Postoperative analgesia, chest physiotherapy were instituted and the patient improved. The patient was also found to have inferior ischial ramus fracture and a closed degloving lower back injury which were all managed in subsequent days in hospital.

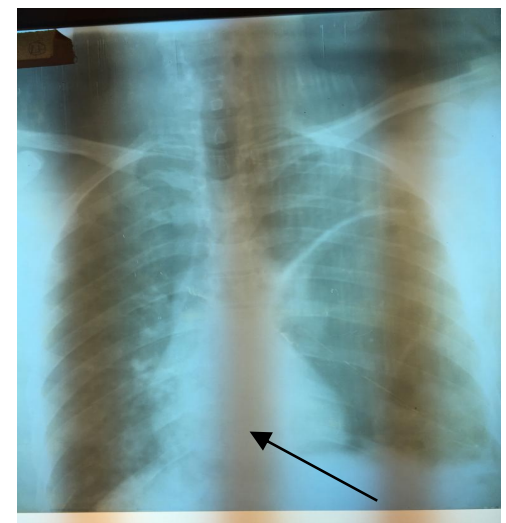

Fig.2: Chest radiography of the patient at the second consultation

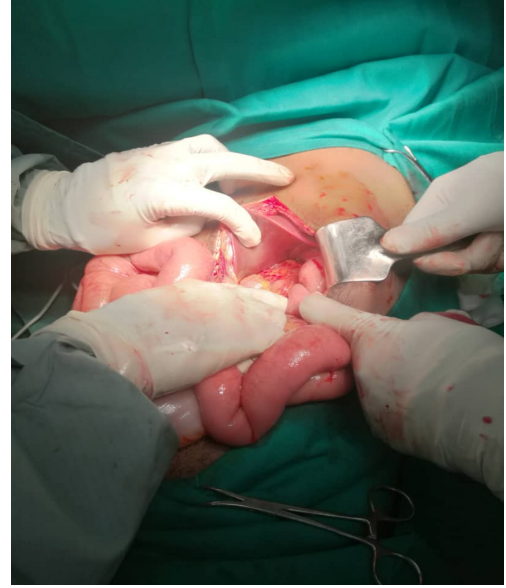

Fig.3: Intraoperative image before reduction of herniated organs

Intraoperative absence of stomach, transverse colon in their normal anatomical location.

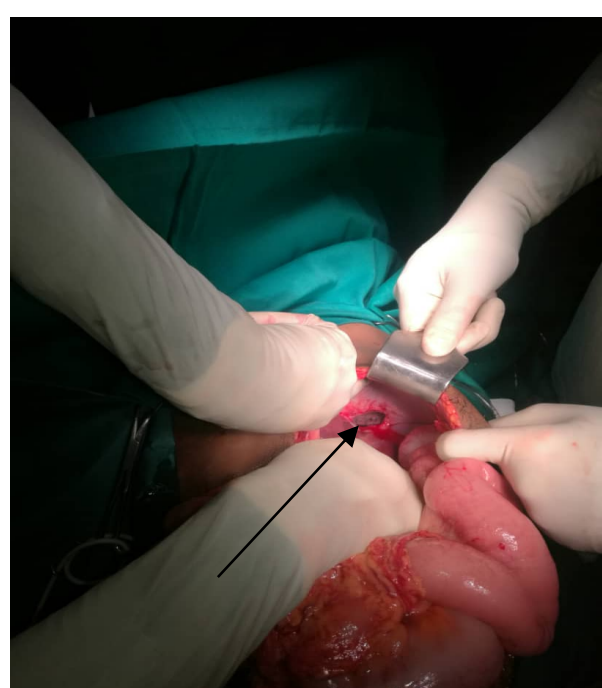

Fig.4: intraoperative image after reduction of herniated organs

A diaphragmatic defect is shown inset after reducing the herniating contents back in the abdomen 


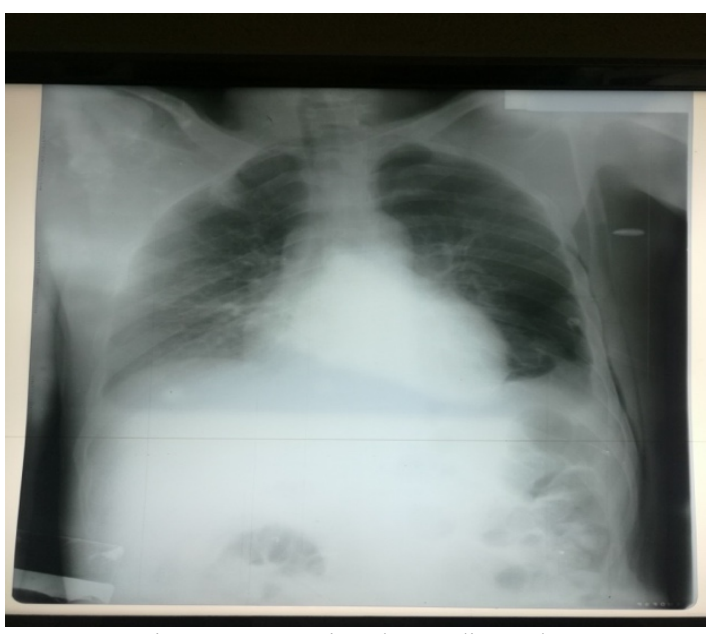

Fig.5: Postoperative chest radiography

Well expanded left lung, mediastinum is central, opacity at the left costo-phrenic angle.

Due to persistent basal opacity on control chest x-ray (Fig 5), the patient was transferred to high facility center for chest CT scan to better define the basal opacity and revealed collapse of left lobe base (Fig 6).

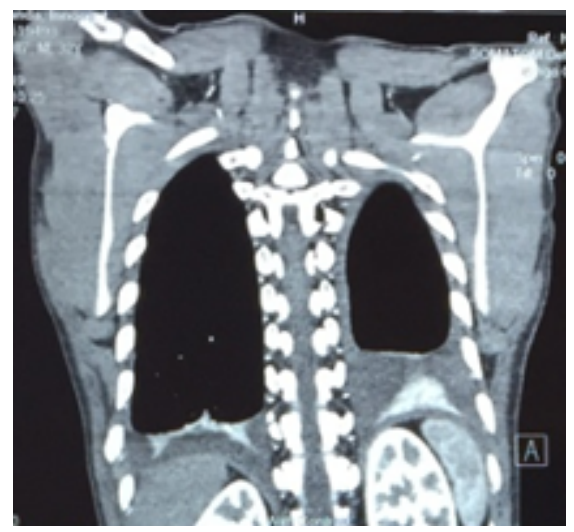

(a)

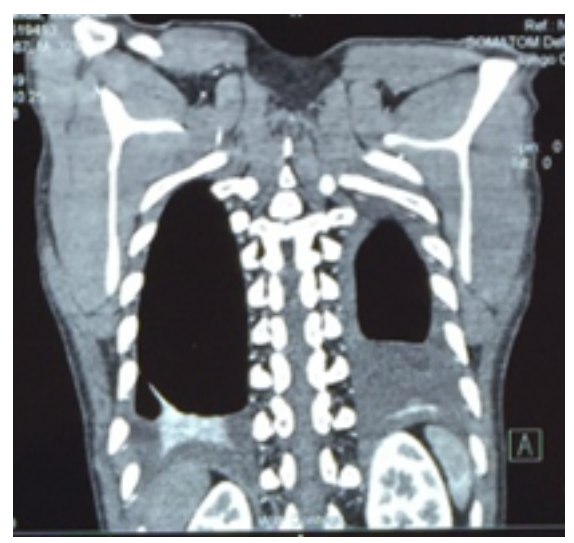

(b)

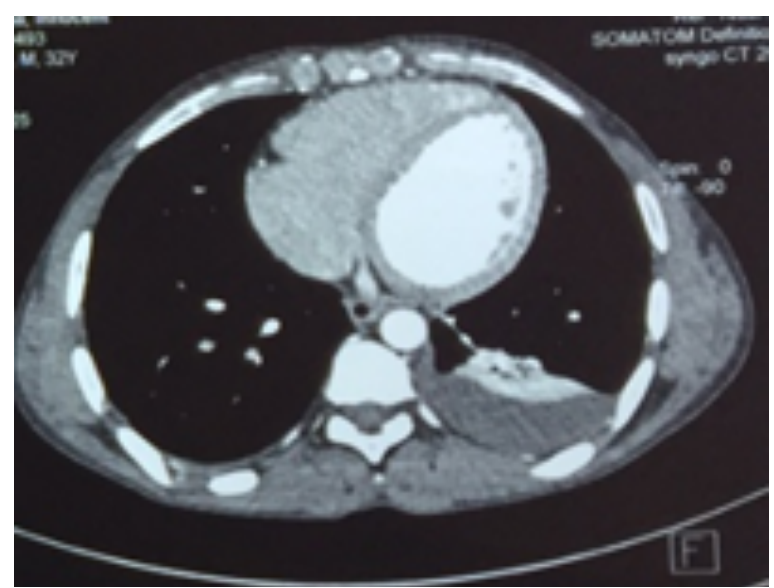

(c)

Fig.6: Chest CT scan of patient presented

Chest CT showing a persistent left basal lobe collapse.

\section{DISCUSSION}

Traumatic diaphragmatic injuries are usually a result of either blunt or penetrating thoraco-abdominal injuries [1].

Diaphragmatic injuries following blunt trauma are often missed compared to penetrating injuries due to low index of suspicion and low likelihood to explore the thoracic or abdominal cavities. Our patient presented to the emergency department few hours after motor vehicle crush with minimal symptoms and then discharged until he presented back with severe respiratory symptoms and initial imaging done was interpreted as pneumothorax instead of viscerothorax. This reveals how difficult it is to diagnose diaphragmatic injuries especially in limited resources settings and low index of suspicion.

When missed the results is either strangulation of hernia contents or tension viscerothorax which are all potentially fatal complications [2]. Our patients presented only with tension viscerothorax without strangulation probably due to the large size of hernia defect.

Tension viscerothorax results from suction of intraabdominal contents into the thorax with herniation of abdominal viscera commonly the stomach but many organs including spleen, kidney, and colon may herniate through the diaphragmatic defect [2]. In our patient only stomach, loops of small bowel, omentum and transverse colon were found in the defect. Increasing occupation of the thoracic cavity with the native abdominal organs displace lung, mediastinal contents and results in kinking of great vessels . Hypoxia and Obstructive shock thus ensue if no emergency treatment is given [5]. Same was found in our patient, however all organs were still viable without perforation.

Whereas in the initial management of tension gastrothorax a nasogastric tube may be inserted to decompress the distended stomach, it commonly posses a technical challenge since the patient is already distressed at the start and might deteriorate further. Failure to decompress the stomach was observed in $14(67 \%)$ of the 25 cases reviewed by Bunya et al but endoscopic decompression was successful in all the cases it was attempted on [6]. At our hospital, endoscopic decompression was not an alternative since it is not available and NGT has failed to decrease the respiratory distress. 
When multiple viscera have herniated into the thoracic cavity, we doubt that the NGT will have any role. Tube thoracostomy and needle thoracostomy have often been used in misdiagnosed cases with poor result and high inherent risk of thoracic contamination [6]. For our case,initial misdiagnosis was immediately recognized by the surgeon who prevented unnecessary insertion of chest tube.

There is paucity of literature regarding ultrasound use in diagnosis of traumatic diaphragmatic hernia. Ultrasound the most easily available imaging modality can detect intra abdominal fluid, intrathoracic bowel loops as well as detect diaphragm motion which may be reduced or abolished in traumatic diaphragmatic hernias [7] [8] [9]. However it is well known to be user dependent and this modality did not contributed much to the diagnosis of diaphragmatic injury for our patient.

Chest x-ray in traumatic diaphragmatic hernia and pneumothorax share a lot in common and this calls for high index of suspicion where CT is not easily available [10]. Reported CT scan sensitivity and specificity is $61-87 \%$ and $72-100 \%$, respectively [11] [12] [13] [14]. However this is not easily available in many rural settings including our hospital. Laparoscopy has a significant role in diagnosis and treatment of diaphragmatic injuries but only limited to hemodynamically stable patients and this advanced technique is not feasible in limited resource settings [15].

Abdominal approach to the hernia versus thoracic approach is dictated by the time between injury and presentation at hospital. In the early post trauma period (less than 6 weeks) abdominal approach is the main stay due to easier access to abdominal organs, ease of reduction as it is assumed that adhesions to lung, mediastinum and pleura haven't formed [16]. The above principle influenced the type of approach we used for our patient but as well considering the easily available surgical instruments at our disposal.

\section{CONCLUSION}

Viscerothorax is an elusive diagnosis that calls for clinical acumen coupled with imaging that has a wide range of sensitivity and specificity. When viscerothorax is missed it can complicate to strangulation of hernia contents or tension viscerothorax which carry a high mortality.

Definitive emergency reduction of herniating contents should be carried out to save the patient's life by either laparotomy or thoracotomy.

\section{REFERENCES}

[1] W. C. Hanna, L. E. Ferri, P. Fata, T. Razek, D. S. Mulder, "The current status of traumatic diaphragmatic injury: lessons learned from 105 patients over 13 years," Ann Thorac Surg, Vol. 85, no. 3, pp. $1044 \mathrm{e} 8,2008$

[2] U. Onakpoya, A. Ogunrombi, A. Adenekan and W. Akerele, "Strangulated tension viscerothorax with gangrene of the stomach in missed traumatic diaphragmatic rupture," ISRN Surgery. doi: $10.5402 / 2011 / 458390.2011$
[3] T. Y. Chern, A. Kwok, and S. Putnis, "A case of tension faecopneumothorax after delayed diagnosis of traumatic diaphragmatic hernia," Surg Case Rep, Vol. 4, pp. 37, Dec 2018

[4] G. J. F. Regalado, M. M. R. Navarro, "Tension gastrothorax as a cause of death by obstructive shock - case report," Rev Chil Pediatr, Vol. 85 , no. 4, pp. 476-480, 2014

[5] R. Shah, S. Sabanathan, A. J. Mearns, and A. K. Choudhury, "Traumatic rupture of diaphragm," Annals of Thoracic Surgery, Vol. 60, no. 5, pp. 1444-1449, 1995

[6] N. Bunya, K. Sawamoto, S. Uemura, T. Toyohara, Y. Mori, K. Ryoko, K. Miyata, H. Irifune, K. Harada and E. Narimatsu, "How to manage tension gastrothorax: a case report of tension gastrothorax with multiple trauma due to traumatic diaphragmatic rupture," International Journal of Emergency Medicine, Vol. 10, no. 4, 2017

[7] E. O. Gerscovich, M. Cronan, J. P. McGahan, K. Jain, C. D. Jones, C. McDonald, "Ultrasonographic evaluation of diaphragmatic motion," J Ultrasound Med, Vol. 20, no. 6, pp. 597-604, Jun 2001

[8] M. Blaivas, L. Brannam, M. Hawkins, "Bedside emergency ultrasound diagnosis of diaphragmatic rupture in blunt abdominal trauma," Am J Emerg Med, Vol 22. No. 7, pp. 601-604, 2004

[9] A. Ammann, W. Brewer, K. Maull, J. Walsh, "Traumatic rupture of the diaphragm: real time sonographic diagnosis" Am J Radiol, Vol. 140 , pp. 915-916, 1983

[10] B. McCann and A. O'Gara, "Tensionviscerothorax: an important deferential for tension pneumothorax," Emergency Medicine Journal, Vol. 22, no. 3, pp. 220-221, 2005

[11] A. Nchimi, D. Szapiro, B. Ghaye, V. Willems, J. Khamis, L. Haquet, "Helical CT of blunt diaphragmatic rupture," Am J Roentgenol, Vol. 182, pp. 24-30, 2005

[12] J. G. Murray, E. Caoili, J. F. Gruden, S. J. Evans, R. A. Halvorsen, R. C. Mackersie, "Acute rupture of the diaphragm due to blunt trauma: Diagnostic sensitivity and specificity of CT," Am J Roentgenol, Vol. 166, pp. 1035-9, 1996

[13] K. L. Killeen, S. E. Mirvis, K. Shanmuganathan, "Helical CT of diaphragmatic rupture caused by blunt trauma," Am J Roentgenol, Vol. 173, pp. 1611-6, 1999

[14] U. K. Bodanapally, K. Shanmuganathan, S. E. Mirvis, C. W. Sliker, T. R. Fleiter, K. Sarada, "MDCT diagnosis of penetrating diaphragm injury," Eur Radiol, Vol. 19, no. 15, pp. 1875-81, 2009

[15] A. A. McDonald, R. H. Bryce, L. Alarcon et al, "Evaluation and management of traumatic diaphragmatic injuries: A Practice Management Guideline from the Eastern Association for the Surgery of Trauma," J Trauma Acute Care Surg, Vol. 85, no. 1, March 2018

[16] P. A. Ebert, R. A. Gaertner and G. D. Zuidema, "Traumatic diaphragmatic hernia," Surgery Gynecology and Obstetrics, Vol. 125 , no. 1 , pp. 59-65, 1967

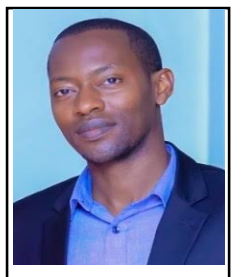

Butana Herbert, born in Mpigi-Uganda, 23/03/1986. Graduated from University of Rwanda in General surgery in 2016. MBChB from Gulu University, Gulu, Uganda, 2011. MMed in general surgery from University of Rwanda, Kigali, Rwanda, 2016.

$\mathrm{He}$ is currently working as Consultant general surgeon at Rwamagana Provincial HospitalRwanda.

Dr Butana is a member of Rwanda surgical society. 